\title{
PROPUESTA DE INTERVENCIÓN DE TRABAJO SOCIAL TERRITORIAL CON COMUNIDADES RURALES DE CHILE CENTRAL EN CONTEXTO DE GLOBALIZACIÓN
}

\section{PROPOSAL FOR TERRITORIAL SOCIAL WORK INTERVENTION WITH RURAL COMMUNITIES OF CENTRAL CHILE IN THE CONTEXT OF GLOBALIZATION}

Fecha recepción: 21 de septiembre de 2020 / fecha aceptación: 29 de diciembre de 2020

Lizbeth Núñez Carrasco ${ }^{1}$

Cómo citar este artículo:

Núñez, L. (2020). Propuesta de intervención de trabajo social territorial con comunidades rurales de chile central en contexto de globalización. Revista Pensamiento y Acción Interdisciplinaria, 6(2), 87-103.

http://doi.org/10.29035/pai.6.2.87

\begin{abstract}
Resumen
Este documento presenta una propuesta metodológica para la intervención de Trabajo Social en comunidades rurales, que mantienen prácticas vinculadas a un modo de vida campesino, cuyos ingresos provienen parcialmente de la agricultura familiar (AFC), que normalmente manejan semillas locales y producen alimentos a distintas escalas. Se presenta el resultado de un proceso de doce años de implementación de dos asignaturas de formación profesional en la carrera de Trabajo Social de la Universidad Católica del Maule, que ha generado vinculación con el medio rural. Bajo un modelo de investigación acción en aula y su correlato in situ, en territorios rurales, los estudiantes organizados en equipos articulan experiencias, conocimientos y debates desarrollados en los cursos, con un proceso de acción en un territorio seleccionado bajo criterios de factibilidad y cercanía. Numerosas experiencias vividas por los estudiantes, en las que construyeron partenariado con actores locales, permitieron levantar información actualizada de la realidad de los productores, e identificar las necesidades de pertinencia en intervenciones sociales. Con todo, se propone un modelo de acción profesional que busca superar las brechas identificadas y orientar su acción a través de objetivos como promover el empoderamiento ciudadano, la soberanía alimentaria y la co construcción de estrategias de inclusión social a distintas escalas, en contexto de desarrollo endógeno. Lo anterior adquiere profundidad, si se considera la relevancia de los productores de alimentos en escenarios de globalización y de crisis, y la importancia de su participación en la gestión de sus territorios. Este documento, primero describe el proceso formativo, luego las características de la población objetivo, y finalmente la propuesta de acción profesional, con sus fundamentos epistemológicos, teóricos y metodológicos. Se espera abrir un debate disciplinar y profesional del Trabajo Social y su vocación de inclusión social.
\end{abstract}

Palabras clave: intervención social, trabajo social, agricultura familiar.

1 Chilena, asistente social Universidad de Chile, Master of teaching in Social Work, The Catholic University of Washington; diplomada en epistemología de las Ciencias Sociales y en Salud Mental, Universidad Santo Tomás, diplomada en Programación Neurolingüística, Universidad Tecnológica Metropolitana. Co investigadora del Proyecto Movilización del patrimonio biocultural en beneficio de la Agricultura Familiar Campesina: vinculando comunidades rurales, Estado y mercado para una agricultura inclusiva. Folio N MEC80190087. Agencia Nacional de Investigación y Desarrollo ANID Resolución exenta N9347/2019. Actualmente, es académica del Departamento de Ciencias Sociales de la Facultad de Ciencias Sociales y Económicas de la Universidad Católica del Maule. Curicó Chile, Correoelectrónico: Inunez@ucm.cl 


\begin{abstract}
This document presents a methodological proposal for the intervention of Social Work with rural communities that maintain practices linked to a farming way of life, and whose incomes partly come from family agriculture. Furthermore, they manage local seeds and produce food at different scales. This work presents the result of a twelve-year process of implementation and connection with the rural environment, which involved two training subjects of the Social Work Program of Catholic University of Maule. Using an action research model that correlated in situ with rural territories, teams of students articulated experiences, knowledge and debates developed in the courses, with a process of action in a territory selected under specific criteria of feasibility and closeness. Numerous students' experiences of building partnerships with local agents, allowed for gathering updated information on the experience of the producers, and identified the needs of appropriateness for social interventions. Using this information, a professional action model is proposed, which searches to overcome the identified gaps and aims at promoting citizen empowerment, food sovereignty, and the co-building of strategies of social inclusion at different scales, within the context of endogenous development. The aforementioned acquires significance considering the relevance of food producers within globalization and crisis scenarios, and the importance of their participation in the management of their territory. This document firstly describes the formative process and the characteristics of the target population, and then proposes professional action with consideration of its epistemological, theoretical and methodological fundamentals. The intention is for a disciplinary and professional debate upon Social Work and its social inclusion vocation to be opened.
\end{abstract}

Keywords: social intervention, social work, family agriculture.

\title{
Presentación
}

Este documento presenta una propuesta de intervención profesional de Trabajo Social con comunidades rurales, que mantienen prácticas socioculturales y económicas vinculadas a un modo de vida campesino, que parte de sus ingresos provienen de actividades de agricultura familiar, normalmente manejan semillas locales y producen alimentos a distintas escalas. En las últimas décadas, han incorporado diversas estrategias como trabajo dependiente en la agroindustria, aunque muchos siguen siendo propietarios o trabajan en mediería. Lo anterior, en contexto de economía de mercado y una política pública en el sector, orientada a convertir al país en un modelo de economía abierta y exportadora de recursos naturales, con el slogan "Chile potencia agroalimentaria". Estas comunidades habitan especialmente en regiones con índices destacados de ruralidad en el centro sur de Chile.

La propuesta es el resultado de un proceso de doce años de implementación de dos asignaturas de formación profesional en la Carrera de Trabajo Social de la Universidad Católica del Maule, Desarrollo Territorial I y II, que se imparten en sexto y séptimo semestre. El modelo resultante fue presentado en el II Congreso Nacional e Internacional de Escuelas de Trabajo Social del CRUCH (Consejo de Rectores de Chile), Antofagasta, Chile 2017, en modalidad ponencia. Ese mismo año el modelo fue puesto a prueba en el Proyecto de investigación internacional, 
"Bases sociales y ecológicas para la gestión participativa de los recursos genéticos de la quinua en comunidades de agricultura familiar campesina de la Región del Maule", MEC folio PAI80160043 (BAQUIANA, 2017-2019), con financiamiento de la Agencia Nacional de Investigación y Desarrollo (ANID), ex Conicyt y del Institut de Recherche pour Developpement (IRD), Francia. Los resultados han sido exitosos para el equipo de investigación y se encuentran en proceso de validación con los actores locales que participaron en el Proyecto. Y cuentan, además, con la validación de dos expertos internacionales, cuyas observaciones han contribuido a esta versión actualizada².

El documento, primero, describe el proceso formativo, la propuesta que articula en bucles dimensiones de investigación acción en aula y su correlato en investigación acción participativa, con comunidades territoriales. Lo anterior, en tiempos de globalización y sus impactos en el ejercicio de ciudadanía (Lechner, 1999), en especial en territorios distintos y/o distantes. Se propone un Trabajo Social capaz de generar allí, en co construcción con los participantes, alternativas actualizadas, apropiadas y pertinentes de acción profesional. Para ello, primero se describe el trabajo de vinculación entre estudiantes universitarios y comunidades; en segundo lugar, se presenta una breve caracterización de la agricultura familiar campesina en Chile. Cada año, se repiten los agradecimientos de los universitarios por las experiencias vividas con estas comunidades, que los reciben de puertas abiertas; el ejercicio pone a prueba, gradualmente, su autonomía profesional, innovación, trabajo de equipo y profesionalismo. En la tercera parte de este documento, se presenta la propuesta de intervención de Trabajo Social con comunidades rurales, especialmente de agricultura familiar campesina y sus fundamentos epistemológicos, teóricos y metodológicos. Se propone ver la acción profesional como un proceso que invita a comunidades a participar y empoderarse de sus territorios, en la constitución de un objeto de cambio y en la configuración de las trayectorias que esperan transitar, poniendo especial énfasis en los valores que orientan el accionar profesional, bajo una relación de cuidado persona-naturaleza.

2 Se agradecen los comentarios y observaciones a este documento por parte del Doctor Gilberto Mascarenhas investigador brasileño independiente asociado al movimiento internacional de investigadores en Sistemas agroalimentarios Localizados, (SIAL), y al Doctor Thierry Winkel investigador del Institut de recherche pour Developpement (IRD), Francia.

Se agradece a la Agencia Nacional de Investigación y Desarrollo (ANID) ex Conicyt por su financiamiento del Proyecto Baquiana, como también al Institut de Recherche pour le Developpement, Francia, por su co financiamiento. 


\section{El proceso socio formativo articulando aula y territorios}

En este apartado, se describe el proceso de trabajo formativo en aula y la aplicación en paralelo de contenidos, competencias, metodologías de Trabajo Social con comunidades en un territorio. Se trata de una experiencia que articula en bucles, constantemente, la reflexión profesional acerca de las experiencias que viven y despliegan in situ, desde las concepciones epistemológicas y teóricas, metodologías y técnicas participativas, en distintos niveles de observación, en aula y territorios. Las concepciones de los participantes, sus construcciones conceptuales, despliegues experimentales y emocionales son fuente de teorización y contrastación, tanto del modelo profesional como de las potencialidades de todos, desde que aceptan el desafío de ponerse en juego, in situ.

A lo largo del proceso, los estudiantes en aula adquieren conocimientos, reflexionan sobre teoría y práctica, experimentan metodologías, analizan modelos, promueven la participación y trabajan en equipo. En paralelo, seleccionan un territorio que será también observatorio (T/O). Inician sus acercamientos, con revisión de documentos, mapas, fotografías, y en el T/O las visitas, para conocer la localidad campesina. Constantemente, definen estrategias y establecen relaciones de colaboración con los actores locales que se interesan por participar en función de sus territorios, con los universitarios. A partir de la configuración conjunta de una situación inicial, desarrollan un proceso de planificación bajo el enfoque estratégico situacional, con el que logran determinar un foco de acción transformadora, una situación final que se espera alcanzar y la trayectoria que articula lo anterior. Se pone énfasis en la generación de conversaciones para lograr acuerdos para la acción, en las que buscan interpretar los intereses de los participantes, y promover compromisos en la ejecución de lo planificado. Se enfatiza en con-versar y no con-vencer. Los equipos despliegan técnicas y procedimientos como entrevistas, asambleas, recorrido comentado, visitas puerta a puerta, entre otros. Se identifican actores clave, líderes, organizaciones sociales, estatales, empresas, etc. Un hito relevante es lograr el establecimiento de un partenariado, que compromete a estudiantes y actores locales a trabajar en pos de sus territorios. En el proceso se articulan percepciones, conocimientos, sensibilidades de actores locales como también información de profesionales y técnicos que trabajan en la comuna; se explora la agenda local y se levanta información que articula Estado, mercado, y sociedad civil, bajo una lógica situacional. El proceso se cierra cuando se ha ejecutado el proyecto co construido, se evalúa y se informa la experiencia vivida.

Al analizar más de 100 experiencias, se constata que la relación entre estudiantes y actores locales se construye gradualmente. Los jóvenes van configurando la relación profesional basada en principios de respeto por las personas, por sus compromisos y por el entorno, con lo que logran generar confianzas y motivar la participación en iniciativas de mutuo acuerdo. Desde los primeros contactos, abriendo tímidamente conversaciones para la acción, hasta 
el cierre del ejercicio de dos semestres, construyen relaciones socio profesionales que hacen posible el logro de objetivos acordados, en medio de dinámicas de cambios que permanentemente experimentan las localidades, en su relación local-global. Al recorrer el territorio, se encuentran con diversas expresiones propias del lugar, características singulares que constituyen parte de su identidad. Estas particularidades, constituyen parte de las riquezas endógenas que hacen única a cada localidad, con una identidad cultural que asombra a los universitarios y promueve sus vínculos. Así, mientras los jóvenes construyen su profesionalidad, desde la comprensión empática y el manejo in situ de procesos de investigaciónacción, en bucles sinérgicos, los actores locales se apropian de los Proyectos y sus proyecciones, renuevan vínculos internos y generan nuevas redes con el entorno público y privado.

A lo largo de años de aplicación de este trabajo, se fueron confirmando los impactos de la globalización en los territorios rurales y en especial se han podido dimensionar las dificultades de políticas y programas sociales orientados a este segmento de la población rural. El Estado en su política sectorial, durante los últimos treinta años, ha puesto énfasis en la habilitación de emprendedores locales. Sin embargo, existen muchos proyectos desde la sociedad civil que requieren mayor protagonismo, es frecuente observar dificultades en la población campesina, para contar con participación empoderada de sus proyectos. Al mismo tiempo, las entrevistas a trabajadores sociales en servicios públicos arrojan el mismo resultado. Los profesionales agradecen poder trabajar con el mundo campesino, reconocen sus saberes locales y la cultura de reciprocidad. Señalan que buscan aplicar los programas sociales con la mayor pertinencia posible. Sin embargo, en lo central se constata que aplican los mismos programas sin distinciones entre realidades rurales, rurbanas y urbanas. En sala se privilegia el debate sobre elementos centrales de los territorios y cómo desplegar procesos innovadores, creativos, y experiencias que contribuyan a fortalecer a las comunidades en sus territorios, promuevan riquezas endógenas y tengan como factor transversal la participación activa de actores locales. En materia de acción pública, una primera constatación es que se mantiene la tendencia a reproducir un Estado centralista y concentrado en las grandes ciudades. Es evidente la necesidad de instrumentos y metodologías apropiadas que establezcan relaciones de cooperación y horizontalidad, y co construyan con las comunidades campesinas desde sus propios intereses, en contextos de planificación pertinentes.

Si bien el desafío se inició asumiendo dos enfoques, primero, el debate acerca de la nueva ruralidad (Grajales \& Concheiro, 2009), y segundo, el enfoque territorial, se constata la necesidad de reflexionar sobre el rol profesional en estos contextos. Las transformaciones que están sufriendo en las últimas décadas los territorios rurales generan escenarios que requieren de nuevas interpretaciones. Es urgente la reivindicación de los actores locales, sometidos históricamente a posiciones desiguales y silenciosas. Es un fenómeno generalizado que impacta, si 
se toma en cuenta que la producción de la AFC a nivel global representa a más del $75 \%$ de la producción de los alimentos que consume la población mundial.

\section{Agricultura familiar campesina (AFC)}

La agricultura familiar es una forma de organizar, la producción agrícola y silvícola, así como la pesca, el pastoreo y la acuicultura, que es gestionada y dirigida por una familia y que en su mayor parte depende de mano de obra familiar, tanto de mujeres como de hombres. La familia y la explotación están vinculadas, co-evolucionan y combinan funciones económicas, ambientales, reproductivas, sociales y culturales (FAO, 2014 en Indap, 2020).

En cifras porcentuales, la agricultura familiar corresponde al $81 \%$ de todas las explotaciones agrícolas de América Latina y el Caribe, ocupa entre el $12 \%$ y el $67 \%$ de la superficie agropecuaria, "y genera entre el $57 \%$ y el $77 \%$ del empleo agrícola en la Región” (Leporati, 2014, en Salcedo y Guzmán ed., p. 36). Aunque estas cifras pueden ayudar a bosquejar la realidad de la AFC en el subcontinente, es importante destacar "que la información existente es disímil y dista de ser comparable entre países" (Leporati et al., 2014, p. 36). La concentración de las explotaciones de agricultura familiar se encuentra en Sudamérica (56\%), otro 35\% en México y las demás en el Caribe (Leporati et al., 2014, p.37). El número de explotaciones dedicadas a las actividades de la agricultura familiar en Sudamérica llega a los 9.205.875, que representa más de la mitad de los 16.596 .837 que existen en la Región. En el Cono Sur, el número de unidades de explotación agrícola es de 6.144.774, de los cuales, más de 5.150 .000 (83\%) son de agricultura familiar. En Chile, estos números son de 301.269 y 277.166 respectivamente. Es decir, la realidad nacional indica que el $92 \%$ de las explotaciones agrícolas desarrollan la actividad de la AF (Leporati et al., 2014, pp. 38). Estos datos contrastan cuando se habla de la distribución de la superficie de la tierra explotable. Un 34,6\% de la superficie agrícola del Cono Sur está ocupada en la agricultura familiar. Son 144.618.362 las hectáreas explotadas por la agricultura familiar en aquel sector del continente, y 11.703 .562 en Chile, lo que representa un poco menos de la mitad de las explotaciones agrícolas en el país (46\%) (Leporati et al., 2014, p. 40)

A fines de 2011, la Organización de Naciones Unidas proclamó el "Año de la Agricultura Familiar" para el 2014, con la finalidad de visibilizar la AFC y otras formas de agricultura de pequeña producción. En el curso de aquel año, se pudo establecer que "... El 88\% de las explotaciones agrícolas del mundo son familiares; que estas proporcionan trabajo a la mayoría de los activos agrícolas; y que representan el principal proveedor de alimentos básicos en los mercados del mundo". (Echenique, 2019, p. 31). De acuerdo a la tendencia de la región, la agricultura familiar, aunque sigue siendo de gran importancia en las actividades económicas del país, ha dejado de ser la principal y única fuente de ingresos para 
los hogares rurales, en provecho de los ingresos provenientes de otras fuentes de trabajo, como la agroindustria. En Chile, según la Encuesta de Caracterización Socioeconómica Nacional (Casen), se considera Agricultura Familiar, "aquellas unidades en las que por lo menos uno de sus miembros definía su ocupación principal como agricultura por cuenta propia". (Casen 2011, en Berdegué, 2014, p. 10). Al hablar de la AF en Chile, Berdegué (2014) define “... agricultura familiar como una forma de organización para la producción agrícola que se basa principalmente en el trabajo de los miembros de un grupo familiar" (p. 9). Como su misma palabra lo dice, es entonces una actividad que se asocia a la existencia de un grupo familiar a partir del cual se emplea a sus miembros y que, por lo tanto, podría generar una relación más estrecha entre el productor y su producto. El autor termina su definición intentando diferenciar implícitamente la agricultura no familiar de la que sí lo es, "independientemente de la forma de tenencia de la tierra, de la superficie de la explotación, o del valor, volumen o destino de la producción". (Berdegué, 2014, p. 9). En el trabajo de Berdegué (2014), en base a los datos del Censo Agropecuario 2007, se define como agricultura familiar un establecimiento en que:

La suma de todos los trabajadores contratados no familiares, a tiempo parcial (estacional) o completo, es igual o inferior a 1 trabajador-equivalente; b) no se emplea un administrador contratado para manejar la explotación; c) no se emplea un administrador no familiar, y; d) no incluyendo explotaciones de propiedad de entidades de gobierno o de la Nación (incluyendo parques nacionales), de colegios e instituciones religiosas, y de otras semejantes. (p. 10).

Siguiendo estos criterios, se pudo establecer que el $71 \%$ de las explotaciones censadas ese año no contrataba ningún trabajador no familiar, y solo en un $13 \%$ declaraban contratar solo a un trabajador-equivalente que no fuera familiar. El número de explotaciones en las que se declaraba contratar a tres o más trabajadores no familiares era apenas de un $8 \%$ en ese censo. De acuerdo al Censo Agropecuario, entonces, la agricultura familiar cuenta en Chile con 219.987 establecimientos dedicados a esta actividad, que se concentran en su mayoría entre las regiones del Biobío y Los Lagos. A lo largo del país, siguiendo la tendencia del continente, se observa una diversificación de las fuentes de ingreso de los trabajadores de la agricultura familiar. En específico, son al menos 125.000 los establecimientos de AF donde al menos tres cuartos de sus ingresos provienen de actividades no agropecuarias. Siguiendo los datos arrojados por la encuesta de Caracterización Socioeconómica de los Hogares (Casen 2011), Berdegué (2014) definió en primer lugar que la unidad a partir de la cual se realiza esta encuesta, es decir, un hogar, era parte de la actividad de la agricultura familiar "cuando al menos uno de sus miembros autodefinía que su ocupación principal era la agricultura por cuenta propia" (р. 9). En ese sentido, la agricultura familiar 
es observada a partir de esta como actividad particular de los individuos, no de los hogares completos. Son hogares en los que la agricultura no es la única fuente de ingresos, entre los miembros que lo componen puede haber quienes viven en un hogar donde se realiza esta actividad, sin dedicarse a ella. Según Berdegué (2014), en el Censo Agropecuario de 2007 se define a la agricultura familiar a partir de la importancia que esta actividad tiene en la generación de ingresos de un hogar y define tres tipos de hogares que practican esta actividad:

Hogares rurales: el aporte de la agricultura por cuenta propia al ingreso total del hogar es inferior a 25\%. b) Agricultura familiar pluriactiva o diversificada: el aporte de la agricultura por cuenta propia al ingreso total del hogar es entre $25 \%$ y $50 \%$.

c) Agricultura familiar especializada: el aporte de la agricultura por cuenta propia al ingreso total del hogar es superior a $50 \%$. (p. 11).

Siguiendo estas características, para la Región del Maule existen 27.728 hogares dedicados a la AF, de los cuales 5.959 tienen una dedicación exclusiva a la explotación de la agricultura familiar y viven de sus ingresos (Censo Agropecuario 2007, en Berdegué, 2014, p.12).

\section{Comunidades territoriales en la Región del Maule}

Luego de revisar los antecedentes estadísticos, en este acápite buscaremos transmitir una descripción en base a las experiencias desarrolladas por estudiantes y docente en innumerables visitas a territorios rurales. Al recorrer las comunidades territoriales en el Maule, se constata la construcción social e identidades culturales que los vinculan. Asimismo, persisten prácticas sociales que se proyectan en el tiempo, y arraigo a costumbres y tradiciones que explican la mantención de estas prácticas, con rasgos de patrimonios. Entre otras, destacan sus expresiones gastronómicas, la biodiversidad agrícola, la flora y fauna que reconocen en sus territorios, las que se asocian a actividades económicas, como también a expresiones artísticas y recreativas. Existe una población frecuentemente envejecida, mayores vulnerables, beneficiarios de pensión básica solidaria, que mantienen huertas familiares como forma de sustento y por tradición. Cabe destacar, el manejo de semillas locales que se encuentran en sus manos por herencia, y por intercambios entre vecinos y familiares. Les preocupa mantener el control de sus semillas, sin embargo, el contexto no es favorable pues los tratados internacionales no consideran a la agricultura familiar y su relevancia en la alimentación de la población. Otro segmento que se asocia a la agricultura familiar, es el de los productores agrícolas, cuyos ingresos provienen principalmente de la explotación de sus predios, generalmente no superiores a 50 hectáreas. Producen hortalizas, frutas y legumbres. Entre sus debilidades presentan brechas en su formación, que les dificultan el acceso a capacitaciones 
técnicas o profesionales, como también a la cultura digital y a las redes de la globalización. Esas brechas también afectan su acceso a créditos "blandos". Al mismo tiempo, las dificultades de acopio, mantención y transporte, por falta de infraestructura y de capital afectan sus precios y disminuyen competitividad, en un entorno donde domina la economía a escala, la agroindustria moderna, competitiva e integrada al mundo. Las distancias que afectan a la agricultura familiar, no solo son físicas por vivir en lugares distantes, sino también se trata de formas de exclusión social que deben ser atendidas con urgencia, si entendemos la importancia de respetar y promover una cultura de producción agroalimentaria sana y a escala local. Es una producción que permite trazar el recorrido desde su origen hasta los consumidores, lo que implica agregación de valor en la cadena agroproductiva. Estas comunidades de productores, llenas de riquezas locales, requieren entornos dinamizadores de sus economías que articulen subsidios, créditos, capacitaciones, accesos a infraestructuras, mejoramiento de sistemas de transporte, cercanía con centros urbanos, entre otros. Internamente, requieren fortalecer sus expresiones de gobernanza ciudadana, empoderarse de sus territorios y participar activamente en su gestión, asegurar su soberanía alimentaria y el control de sus riquezas locales.

\section{La propuesta: Trabajo Social territorial y comunitario}

El objetivo central de la propuesta es la inclusión socioterritorial de las comunidades en la relación local global, y apunta a la complejización de territorios que son concebidos en cuanto espacios geográficos socialmente construidos, que se constituyen como el lugar social de la vida, cuya historia y cultura en interacción con su entorno les brinda un "lugar en el mundo" a sus habitantes. En este sentido (Cuervo, 2006; Boisier, 2009), un territorio es "ganador" cuando es capaz de lograr una estructura territorial interna de una complejidad comparable a la exhibida por el espacio de la globalización, para un acoplamiento dinámico en su núcleo y no en la periferia. Por ello, se propone que el Trabajo Social apunte a generar formas de inclusión, en co construcción, desde niveles locales hacia escenarios globales, en perspectiva estratégica. Es así, que los profesionales participan en definiciones epistémicas y en planificación situacional, e instalan procesos dialógicos y acciones vinculantes que procuran visibilizar, recuperar y articular, desde la dignidad de ls personas y sus comunidades, el control de sus vidas y sus territorios.

Se entiende a la globalización en términos de capitalismo financiero y de comunicaciones (Castell, 2000; Cuervo, 2006). Uno de los debates desarrollado en las Ciencias Sociales, se refiere a los efectos homogenizadores que este fenómeno tendría en las economías y culturas locales. Efectivamente, los avances de la tecnología están ampliando la cobertura, y llegando con energía e información a lugares remotos y aislados. En este punto, cobran especial relevancia las dimensiones que fundamentan intervenciones en Trabajo Social, tales como sentido de identidad, arraigo y pertenencia de las comunidades con sus territorios; 
la relación sociedad naturalezay, fundamentalmente, la ingenteparticipación de sus habitantes en las decisiones importantes de la vida en común. Las experiencias se articulan en la configuración de un Proyecto territorial. Estudiantes, comunidades y profesores triangulan el proceso hasta llegar a la provocación de un cambio socio cultural. El proceso pone en juego al académico(a) que modela y da seguimiento, a los estudiantes en despliegue profesional creciente, y a comunidades llamadas a empoderarse. Cabe destacar que el valor fundamental de esta propuesta es la vida, en todas sus expresiones, por lo tanto, las dimensiones de justicia socioterritorial y sustentabilidad ambiental (Vandana, 2011) son fundamentales. Las evidencias de cambio in situ son experiencias interpretadas y co construidas, relatos, videos, fotografías, informes, etc.

\section{Referentes éticos, epistemológicos y teóricos}

En términos de ética, la propuesta desarrolla una constante reflexión inspirada en principios de humanismo cristiano y de doctrina de los derechos humanos, con una opción democrática, ecológica, y con énfasis en el cuidado de las personas y la tierra (Boff, 2002).

Epistemológicamente, esta propuesta opta por el constructivismo que asume la integración de la realidad social y del sujeto-objeto, en una síntesis que reúne el conocer y el actuar, como dos caras de una misma moneda (Bozzano, 2009). Lo anterior ocurre cuando se integran saberes locales, conocimiento científico e institucional, en construcción dialógica buscando instalar procesos de toma de decisiones participativos. Entre los principios del constructivismo que se privilegian, para movilizar las experiencias destaca el valor del aprender haciendo, de la relación teoría práctica y en especial la valoración de los conocimientos previos de los sujetos. Destaca entre sus postulados, que la realidad social es construida a través del lenguaje; lo que sabemos, creemos y constatamos es fruto del lenguaje con el que interpretamos y comprendemos la realidad. Esta corriente, en Educación, postula que todos los seres humanos aprendemos a lo largo de toda la vida y que quien organiza, analiza, sistematiza y concluye, construye el conocimiento. El conocimiento se construye en la interacción social frecuente y se produce cuando es significativo para quien aprende. Desde el punto de vista teórico, la propuesta opta por un enfoque eco sistémico y territorialista (Boisier, 1999; FAO, 2017) que busca dar cuenta de la complejidad social (Morin, 1994). Lo anterior, desde lecturas construidas con los expertos, sus habitantes, bajo un enfoque de planificación estratégica y situacional (Matus, 1972).

Con base en el conocimiento científico y comprensión empática de las situaciones sociales, el Trabajo Social incorpora energía e información en los territorios. Se busca interpretar la complejidad territorial (Bozzano, 2009) sobre las situaciones sociales y allí definir sujeto-objeto. Esto supone incorporar en los análisis el valioso relato de los habitantes, "lo cualitativo", el mundo de la vida, como también el dato preciso de la ciencia, "lo cuantitativo" y la información pertinente 
y actualizada de las instituciones públicas. Por ello, la tríada Estado, mercado y sociedad civil, debe ser un eje permanente del marco analítico. Al Trabajo Social le corresponde potenciar miradas complejas que asuman la dinámica local global, como también gestionar conocimiento e información, redes, diagnósticos territoriales, innovar, considerando el impacto de la globalización (Parola, 2001) en la acción local, y movilizar recursos. Los desafíos apuntan a fortalecer potencialidades, construir redes, crear espacios de diálogo, democratizar el tejido social y el conocimiento, manejar conflictos, visibilizar realidades. Los habitantes de los territorios-dueños por herencia, de sus geografías- son los encargados de decidir sobre el pasado, presente y futuro de sus realidades.

Con todo, se propone que la acción profesional se oriente a instalar procesos de inclusión en dignidad, de lo local en el núcleo de lo global. Lo anterior, con base en la activación de procesos psico sociales y socioculturales, que reivindiquen y recuperen los valores de las identidades locales y sus patrimonios. Aquí, el protagonismo lo tienen los habitantes, el trabajador(a) social aporta conocimientos, redes, información, capacitación, y especialmente, comprensión empática, conversaciones para la acción, construcción de confianzas y alianzas estratégicas. Un componente fundamental en el proceso se refiere a las restituciones con las comunidades. Es primordial constrastar lo que estamos interpretando, si estamos representando a los actores en juego, a sus intereses, preocupaciones, sensibilidades, visiones, prospecciones.

En términos de docencia, la experiencia se desarrolla, durante dos semestres, en que se despliega el diseño y ejecución de la investigación acción educativa en aula, en concomitancia con procesos de investigación acción in situ, con diversos grados de participación comunitaria. Es un proceso en bucles, cada vez que se recrea, surgen nuevos desafíos, que ponen en juego el debate entre universitarios y actores locales, con el seguimiento constante de docentes.

\section{Referentes Metodológicos}

En términos metodológicos, se articula la experiencia formativa de estudiantes avanzados de la carrera y acciones profesionales en territorios con sus comunidades. Ambas dimensiones en bucle se retroalimentan constantemente, contrastando teoría y práctica, dimensiones cualitativas y cuantitativas, se construye en conjunto un relato del territorio, de sus prioridades y sensibilidades, base de los acuerdos para la acción, como también para su planificación, ejecución y evaluación. La opción de planificación es situacional y estratégica. A continuación se presentan los pasos que se desarrollan en paralelo en aula y en territorios: 
En aula

-) Libre constitución de equipos de trabajo de estudiantes, distribución de poder y protagonismo. Entrenamiento en la noción de equipo profesional, incluyendo destrezas en conducción, comunicación, y habilidades comunicacionales.

-) Selección del territorio observatorio (T/O) bajo criterios pre establecidos.

-) El equipo comienza a trabajar en el aula, y en acercamientos sucesivos al (T/O).

-) Se avanza en crecientes grados de cientificidad social en diálogo con la realidad.

-) Aplicación de metodologías y técnicas participativas. Se desarrollan en sala hitos, se comparten y analizan estados de avance, evaluaciones de proceso, y constantes ejercicios con base en metodologías participativas.

-) Acompañamiento docente constante como así mismo de pares en retroalimentación, entendiendo que se trata de profesionales en formación avanzada.

\section{En territorios}

-) Acercamientos sucesivos a los territorios, contacto con Estado, mercado y sociedad civil. Construcción de vínculos y confianzas.

-) Caracterización de la unidad de estudio. En esta etapa es central el diálogo y una actitud ética constante, de confianza.

-) La etapa de inserción, contacto y acuerdos permite el conocimiento de ubicación espacial, que debe incluir mapa del ecosistema, demografía, morbilidad, servicios sociales. De particular relevancia, la cultura, valores, costumbres, la relación sociedad - naturaleza las riquezas de su biodiversidad y paisajes.

En esta etapa se logra identificar, analizar y describir en perspectiva etnográfica, dimensiones fundamentales como la estructura económica que le da sustento al territorio y su relación con la economía global.

-) Construcción de diagnósticos situacionales, y determinación de la situación deseada. Determinación de objetivos; elaboración de estrategias; elección de los medios.

-) Ejecución y evaluación de las acciones planificadas en partenariado.

-) Presentaciones finales, una inflexión central donde se juegan competencias profesionales en complejidad creciente: traemos el T/O al aula. 


\section{Algunos resultados}

Algunos resultados que se han logrado documentar en las experiencias territoriales son: el fortalecimiento de organizaciones vecinales, activación de redes con el sector público y con privados, fortalecimiento de costumbres territoriales, visibilización de historias locales a través de exposiciones, ferias locales, textos redactados en conjunto entre universitarios y actores locales, gestión y articulación de recursos humanos, articulación de productores locales con redes de comercialización, identificación y promoción de productos identitarios, entre otros.

Se presenta una experiencia resumida que ilustra en parte la aplicación del Modelo. Un equipo de estudiantes decidió trabajar en la localidad de Cunaco, Región de O’Higgins; una de sus integrantes es nativa y desarrollaba su práctica profesional en un Centro de Salud Familiar local. Esto, facilitó al equipo, la inserción y aplicación de técnicas como observación, entrevistas, escucha activa, focus group, entre otros. Según sus apreciaciones, esto, les permitió conocer la cultura, costumbres, parte del patrimonio local, pero principalmente a las personas que habitan en este lugar y generar diálogos, mediante el recorrido por el territorio.

Esta etapa de conocimiento y generación de confianzas culminó con el establecimiento de compromisos entre las estudiantes y una agrupación de emprendedores locales, que se encontraba sin actividad y se motivó por planificar en conjunto la instalación de una Feria de artesanías locales. El modelo territorial propicia las articulaciones Estado, mercado y sociedad civil; en este caso, gracias a estas coordinaciones, los artesanos obtuvieron permiso, espacio, difusión y todo lo necesario para instalar la Feria. El equipo de profesionales en formación, en conjunto con la agrupación de emprendedores, elaboró una estrategia situacional con el fin de generar una transformación social desde los mismos actores involucrados.

Con esta intervención también se esperaba generar visibilidad y valoración de las artesanas en la comunidad y su entorno regional. Y generar ingresos, ya que gran parte de las mujeres son jefas de hogar, que al realizar esta actividad no solo están generando ingresos económicos, sino que además crean instancias de conversación, valoración, participación entre los vecinos, desarrollando y fomentando el trabajo en equipo. Finalmente, este equipo generó negociaciones exitosas, dejando la Feria instalada y con las autorizaciones necesarias, apuntando a la sustentabilidad del proyecto en el tiempo.

Cabe recordar que actualmente nos encontramos en el proceso de validación comunitaria respecto de los resultados de la aplicación de este modelo de intervención, con el Proyecto de investigación internacional, "Bases sociales y ecológicas para la gestión participativa de los recursos genéticos de la quinua en comunidades de agricultura familiar campesina de la Región del Maule", MEC folio PAI80160043 (Baquiana, 2017-2019), con financiamiento ANID ex Conicyt. Este proceso se encuentra detenido por la crisis ocasionada por la pandemia de Covid-19 y se espera retomar en cuanto las restricciones sanitarias lo permitan. 


\section{Conclusiones y proyecciones para el Trabajo Social}

El Trabajo Social promueve desde sus fundamentos el valor de la vida, el bien común y la solidaridad, que garanticen la paz social, la inclusión y el respeto irrestricto a los derechos humanos, el trato digno a cada persona y a cada ser vivo, en contextos socialmente justos y ambientalmente sustentables. Destaca el principio de autodeterminación de cada ser humano en lo que más le concierne, en su propia vida. Es fundamental el derecho de participación en la construccion del presente, lecturas del pasado y proyecciones futuras.

Con todo, se trata de colaborar en la construcción de niveles de participación decisionales, más allá de los puramente consultivos o informativos. Y de instalar procesos dialógicos democratizadores, en el máximo de escenarios posibles, a distintas escalas, poniendo las realidades y las culturas locales en el centro de las conversaciones para la acción, en la díada conocer/actuar. Este efecto solo es posible bajo un tratamiento respetuoso de las iniciativas que surgen en los diálogos entre universitarios y habitantes locales, con constante acompañamiento docente que respeta y apoya la autonomía creciente de los actores en juego. Las experiencias se desarrollan en especial con comunidades de agricultura familiar campesina, una población especialmente excluida en lo que va del siglo XXI. Aquí se abre entonces un conjunto de desafíos acerca de la realidd de la producción de alimentos sanos, bajo principios de comercio justo y con sistemas de regulaciones que garanticen el ejercicio de los derechos ciudadanos de quienes hoy producen los alimentos. Con esta propuesta de acción profesional de Trabajo Social con comunidades en territorios rurales, esperamos ser un aporte en la configuración de nuevos estadios de desarrollo en dignidad y rescate, en y desde los pueblos, culturas y saberes locales, para promover desde sus fuerzas endógenas, sus proyectos de futuro, de tal suerte que puedan aspirar y alcanzar formas de inclusión desde las periferias actuales hacia los centros globalizados y se instauren procesos de comercialización justos y sustentables. Ello, con base en sus multiples talentos y vocaciones, en sus herencias acerca de lo que son, de sus formas de vivir y de lo que quieren y sueñan para sus futuros. 


\section{Referencias bibliográficas}

Arévalo, J. M. (2004). La Tradición, el patrimonio y la identidad. Revista de estudios extremeños, 60(3), 925-956. Recuperado de: https://www.dip-badajoz.es/ cultura/ceex/reex_digital/reex_LX/2004/T.\%20LX\%20n.\%203\%202004\%20 sept.-dic/RV000002.pdf

Berdegué, J.A. (2014). La Agricultura Familiar en Chile, Serie Documento de Trabajo N 152, Grupo de Trabajo Desarrollo con Cohesión Territorial, programa Cohesión Territorial para el Desarrollo. Santiago de Chile: Rimisp. Recuperado de: https://www.indap.gob.cl/docs/default-source/descargas-agriculturafamiliar-campesina/rimisp_2014_griculturafamiliarenchile.pdf?sfvrsn=2

Boff, L. (2002). El cuidado esencial, ética de lo humano, compasión por la tierra. Madrid: Trotta. Recuperado de: https://redmovimientos.mx/wp-content/ uploads/2020/07/El-Cuidado-Esencial-Boff.pdf

Bosier, S. (1999). Teoría y Metáforas sobre el Desarrollo Territorial. Santiago de Chile: Cepal. Recuperado de: https://repositorio.cepal.org/bitstream/ handle/11362/2189/1/S9860432_es.pdf

Bozzano, H. (2009). Territorios: El método territorii. Una mirada territorial a proyectos e investigaciones no siempre territoriales. 8th International Conference of Territorial Intelligence, llevado a cabo en la ciudad de Salerno, Italia. Recuperado de: https://halshs.archives-ouvertes.fr/halshs-00533337/ document

Bozzano, H. (2005). Territorio y Gestión. Conocimiento, realidad y transformación: un círculo virtuoso. VII Encuentro Internacional Humbolt, llevado a cabo en Merlo, Argentina. Recuperado de: https://scholar.google.com/scholar?cluste $r=3545997796675100244 \& h \mid=e n \& o i=s c h o l a r r$

Castell, M. (2000). Globalización, sociedad y política en la era de la información. Bitácora Urbano-Territorial, 4(1), 42-53. Recuperado de: https://dialnet. unirioja.es/servlet/articulo?codigo $=4008342$

Concheiro Bórquez, L., \& Grajales Ventura, S. (2009). Nueva ruralidad y desarrollo territorial. Una perspectiva de los sujetos sociales. Veredas Revista de Pensamiento Sociológico, (18), 145-167. Recuperado de: https://publicaciones.xoc.uam.mx/resumen_articulo.php?id=5905

Cuervo, L. M. (2006). Globalizacióny Territorio. Santiago de Chile: Cepal. Recuperado de: https://repositorio.cepal.org/bitstream/handle/11362/7315/S0600224_ es.pdf?sequen

Echenique, J. (2019). Evolución de la economía campesina en América Latina. En M. I. Fernández (Ed.), Perpectivas para el Desarrollo Rural Latinoamericano. 
Un homenaje a Alexander Schejtman. (pp. 31-80). Buenos Aires: Teseo. Recuperado de: https://www.editorialteseo.com/archivos/16544/ perspectivas-para-el-desarrollo-rural-latinoamericano/

Asociación de Cooperativas Vitivinícolas Argentinas, ACOVI (2013). Agricultura familiar en cifras. Recuperado de: http://acovi.com.ar/observatorio/wpcontent/uploads/2014/06/agricultura-familiar-en-cifras.pdf

Instituto de Desarrollo Agropecuario Indap (2020). Agricultura Familiar Campesina. Recuperado de: https://www.indap.gob.cl/te-recomendamos/ agricultura-familiar-campesina\#: :text=En\%20el\%20marco\%20del\%20 A\%C3\%B1o,una\%20familia\%20y\%20que\%20

Lechner, N. (2000). Nuevas ciudadanías. Revista de estudios sociales, (5), 25-31. Recuperado de: https://www.redalyc.org/pdf/815/81500504.pdf

Lechner, N. (1999). Las condiciones sociopolíticas de la ciudadanía. IX Curso Interamericano de elecciones y democracia. Instituto Interamericano de Derechos Humanos Capel, Instituto Federal Electoral. Ciudad de México. Recuperado de: http://www.desarrollohumano.cl/Extension/Discursos/ capel.pdf

Leporati, M., Salcedo, S., Jara, B., Boero, V., \& Muñoz, M. (2014). La agricultura familiar en cifras. En Salcedo S. y Guzmán, L. (Eds.) Agricultura Familiar en América Latina y el Caribe: recomendaciones de Política. (pp. 35-56). Santiago de Chile: FAO. Recuperado de: http://www.fao.org/3/i3788S/i3788S.pdf

Matus, C. (1972). Política Planificación y Gobierno. Caracas: Fundación Altadir, ILPES. Recuperado de: http://www.trabajosocial.unlp.edu.ar/uploads/ docs/2_carlos_matus_politica__planificacion_y_gobierno_.pdf

Morin, E. (1994). Introducción al Pensamiento Complejo. Recuperado de: http://cursoenlineasincostoedgarmorin.org/images/descargables/Morin_ Introduccion_al_pensamiento_complejo.pdf

Morin, E. (1999). Los siete saberes necesarios para la educación del Futuro. Recuperado de: https://www.uv.mx/dgdaie/files/2012/11/CPP-DC-MorinLos-siete-saberes-necesarios.pdf

Organización de las Naciones Unidas para la Alimentación y la Agricultura, FAO. (2007). El enfoque ecosistémico aplicado a la Alimentación y la Agricultura: Situación y Necesidades. Recuperado de: http://www.fao.org/forestry/130290c7da1b003c228e3de319d89da3264977.pdf

Parola, R.N. (2001). Acción colectiva e intervención profesional. Consideraciones teóricas- metodológicas a propósito del Trabajo Social Comunitario. Boletín 
Sura $N^{\circ}$ 59, julio, Escuela de Trabajo Social, Universidad de Costa Rica. Recuperado de: http://www.ts.ucr.ac.cr/binarios/sura/sura-0059.pdf

Pérez, E. (2001). Haciauna nuevavisión delorural. En N. Giarraca, (comp.)¿ Una Nueva ruralidad en América Latina? (pp. 17-29). Buenos Aires: Clacso. Recuperado de: http://biblioteca.clacso.edu.ar/clacso/gt/20100929011414/2perez.pdf

Rogers, C. (1992). El Proceso de convertirse en persona. Mi técnica terapéutica. Recuperado de: https://jesuitas.lat/uploads/el-proceso-de-convertirseen-persona/CARL\%20ROGERS\%20-\%201992\%20-\%20EL\%2OPROCESO\%20 DE\%20CONVERTIRSE\%20EN\%2OPERSONA.pdf

Salcedo, S., \& Guzmán, L. (Ed.). (2014). Agricultura Familiar en América Latina y el Caribe. Serie Recomendaciones de Política. Santiago de Chile: FAO. Recuperado de: http://www.fao.org/3/i3788s/i3788s.pdf

Schejtman, A., \& Berdegué, J. (2004). Desarrollo Territorial Rural. Santiago de Chile: Rimisp. Recuperado de: https://www.rimisp.org/wp-content/ files_mf/1363093392schejtman_y_berdegue2004_desarrollo_territorial_ rural_5_rimisp_CArdumen.pdf

Schneider, S. (2014). Informe síntesis: La Agricultura Familiar en América Latina. Un nuevo análisis comparativo. Recuperado de: https://www.ifad.org/ documents/38714170/39135645/Family+farming+in+Latin+America++A+new+comparative+analysis_s.pdf/9330a6c4-c897-4e1c-9c051144ebec0457

Shiva, V. (2011). Democracia de la Tierra y los Derechos de la Naturaleza. Instituto de Estudios Ecologistas del Tercer Mundo. Conferencia llevada a cabo en la ciudad de Quito, Ecuador. Recuperado : https://docplayer.es/14535276Conferencia-magistral-democracia-de-la-tierra-y-los-derechos-de-lanaturaleza-vandana-shiva-quito-26-de-noviembre-2011.html

Weinstein, L. (2004). La espiritualidad y el yo como bases de una militancia en la vida. Polis 8, 1-12. Recuperado de https://journals.openedition.org/ polis/6075

Dirección de correspondencia:

Lizbeth Núñez Carrasco

Contacto: Inunez@ucm.cl

Esta obra se encuentra bajo una Licencia de Creative Commons

Reconocimiento-NoComercial-Compartirlgual 4.0 Internacional

Escuela de Trabajo Social · Universidad Católica del Maule 